\title{
Cross-study analysis of gene expression data for intermediate neuroblastoma identifies two biological subtypes Patrick Warnat ${ }^{1}$, André Oberthuer ${ }^{2}$, Matthias Fischer ${ }^{2}$, Frank Westermann ${ }^{3}$, Roland Eils ${ }^{1}$ and Benedikt Brors*1
}

\author{
Address: ${ }^{1}$ Department of Theoretical Bioinformatics (B080), German Cancer Research Center (DKFZ), Im Neuenheimer Feld 280, D-69120 \\ Heidelberg, Germany, ${ }^{2}$ Children's Hospital, Department of Pediatric Oncology and Hematology and Center for Molecular Medicine Cologne \\ (CMMC), University of Cologne, Kerpener Strasse 62, D-50924 Cologne, Germany and ${ }^{3}$ Department of Tumor Genetics (B030), German Cancer \\ Research Center (DKFZ), Im Neuenheimer Feld 280, D-69120 Heidelberg, Germany \\ Email: Patrick Warnat - pwarnat@web.de; André Oberthuer - andre.oberthuer@uk-koeln.de; Matthias Fischer - matthias.fischer@uk-koeln.de; \\ Frank Westermann - f.westermann@dkfz-heidelberg.de; Roland Eils - r.eils@dkfz.de; Benedikt Brors* - b.brors@dkfz.de \\ * Corresponding author
}

Published: 25 May 2007

BMC Cancer 2007, 7:89 doi:10.1/86/147/-2407-7-89
Received: 21 December 2006

Accepted: 25 May 2007

This article is available from: http://www.biomedcentral.com/I47/-2407/7/89

(c) 2007 Warnat et al; licensee BioMed Central Ltd.

This is an Open Access article distributed under the terms of the Creative Commons Attribution License (http://creativecommons.org/licenses/by/2.0), which permits unrestricted use, distribution, and reproduction in any medium, provided the original work is properly cited.

\begin{abstract}
Background: Neuroblastoma patients show heterogeneous clinical courses ranging from lifethreatening progression to spontaneous regression. Recently, gene expression profiles of neuroblastoma tumours were associated with clinically different phenotypes. However, such data is still rare for important patient subgroups, such as patients with MYCN non-amplified advanced stage disease. Prediction of the individual course of disease and optimal therapy selection in this cohort is challenging. Additional research effort is needed to describe the patterns of gene expression in this cohort and to identify reliable prognostic markers for this subset of patients.
\end{abstract}

Methods: We combined gene expression data from two studies in a meta-analysis in order to investigate differences in gene expression of advanced stage ( 3 or 4 ) tumours without MYCN amplification that show contrasting outcomes (alive or dead) at five years after initial diagnosis. In addition, a predictive model for outcome was generated. Gene expression profiles from 66 patients were included from two studies using different microarray platforms.

Results: In the combined data set, 72 genes were identified as differentially expressed by metaanalysis at a false discovery rate (FDR) of $8.33 \%$. Meta-analysis detected 34 differentially expressed genes that were not found as significant in either single study. Outcome prediction based on data of both studies resulted in a predictive accuracy of $77 \%$. Moreover, the genes that were differentially expressed in subgroups of advanced stage patients without MYCN amplification accurately separated MYCN amplified tumours from low stage tumours without MYCN amplification.

Conclusion: Our findings support the hypothesis that neuroblastoma consists of two biologically distinct subgroups that differ by characteristic gene expression patterns, which are associated with divergent clinical outcome. 


\section{Background}

Neuroblastoma is a malignant tumour of the sympathetic nervous system. Next to brain tumours, it is the most common solid tumour in children, with 7.5 cases per 100,000 infants [1]. The hallmark of this tumour is its heterogeneous clinical behaviour, ranging from life-threatening tumour progression to spontaneous regression or differentiation to benign ganglioneuroma. To discriminate these contrasting patterns of clinical behaviour, several molecular and cytogenetic features, such as amplification of the MYCN oncogene or deletion of chromosomal material from $1 \mathrm{p}$ or $11 \mathrm{q}$ are currently used in clinical trials $[1,2]$. However, the course of intermediate risk patients with MYCN non-amplified advanced stage disease is still hard to predict by these markers, and optimal therapy selection in this cohort remains challenging. Thus, current neuroblastoma trials may stratify these advanced stage MYCN non-amplified patients to either the low-, the intermediate- or the high-risk group leading to highly differing treatment approaches [3]. Therefore, additional research effort is needed to identify reliable prognostic markers for these subsets of patients [4].

To this end, recent studies applied gene expression profiling to investigate divergent clinical neuroblastoma phenotypes [5-8]. These studies have shown that it is possible to discriminate subtypes of neuroblastomas of diverse molecular and clinical phenotype by their gene expression profiles. However, gene expression data for the important subgroup of MYCN non-amplified neuroblastoma patients of advanced stage disease (International Neuroblastoma Staging System, INSS Stage 3 or 4,) is still rare, leading to a high risk of false positive and false negative findings [9].

In this study, we combined gene-expression data from two different studies generated by different platforms, since a combined analysis yields more information than each individual study $[10,11]$. Although microarray data generated by different platforms are not directly comparable, they can be combined in an integrative analysis, if appropriate methods are carefully chosen [12]. We focused on MYCN non-amplified tumours with advanced INSS stage 3 or 4 . We searched for differences in gene expression between patients with contrasting outcomes (alive or dead) at five years after initial diagnosis. Differentially expressed genes were identified and described based on data of the two largest neuroblastoma gene expression studies to date $[7,8]$. In addition, a predictive model (classifier) for patient outcome was generated and assessed.

\section{Methods \\ Gene Expression Data}

Microarray data by Ohira et al. [7] were downloaded from the NCBI GEO [13] database (accession number
GSE2283), and microarray data by Oberthuer et al. [8] were downloaded from the EBI ArrayExpress [14] database (accession number E-TABM-38). For both data sets normalized expression data were used as published.

The probes of the different microarray platforms were mapped by a perfect match of their sequences. If an oligonucleotide probe of the platform used by Oberthuer et al. had a sequence that could be matched to a part of the GenBank mRNA sequence corresponding to a cDNA clone probe of the platform used by Ohira et al., both probes were considered as representing the same transcript. For genes represented by several probes on a platform the median expression value of all corresponding probes was used.

Gene expression profiles of patients were selected from both studies according to the following criteria: MYCN non-amplified tumors with advanced INSS stage (3 or 4) with either a minimum follow-up time of five years after diagnosis or with fatal outcome of disease. All patients were grouped according to their outcome status (alive vs. dead) five years after initial diagnosis. In addition, we randomly selected 40 other patients from both studies (MYCN amplified stage 3 or 4 tumours, ten of each study; MYCN non amplified tumours with stages 1, 2 or 4 S, ten of each study). According to their clinical characteristics, these patients have either a highly aggressive neuroblastoma tumour or a tumour with very good prognosis. We used the microarray data of these tumors to study the expression of the genes found to be differentially expressed in our meta-analysis of intermediate risk neuroblastomas in patients with either distinct favourable or unfavourable course of disease.

\section{Identification of significant genes}

To detect significant differential expression of a gene between the two outcome patient groups across studies, we applied a meta-analysis approach as described by Choi et al. [10]. The Bioconductor [15] software package GeneMeta was used to perform these calculations.

Moreover, to explore the biological aspects of the significant genes, we analysed the associated Gene Ontology (GO) [16] terms using the GOstat software [17], which generates statistics of which annotations are overrepresented in a given list of genes.

\section{Classification analysis}

A predictive model for the patient outcome status (alive vs. dead) five years after diagnosis was generated using the method of nearest shrunken centroids classification [18].

Using only the data of genes represented on both microarray platforms $(\mathrm{n}=1,271)$, we applied a methodology 
evaluated by Warnat et al. [11], namely the median rank scores (MRS), to derive numerically comparable quantities from the expression values of both platforms used in the different studies. In total, 36 patients were selected from both studies as a training set to generate a predictive model of patient outcome (Additional File 1). The remaining 30 patients were used as an independent test set. In addition, samples of the independent test set were classified according to the clinical markers age at diagnosis and INSS stage.

Supplementary information is available at the website of BMC Cancer.

\section{Results Gene Expression Data}

We focused on data of the two biggest current neuroblastoma gene-expression studies from Ohira et al. [7] and Oberthuer et al. [8]. These studies analysed a total of 136 (Ohira) and 251 patients (Oberthuer). Further neuroblastoma gene expression studies by Wei et al. [5] and Schramm et al. [6] comprised 18 and 4 patients that met the criteria of our cohort (patients with advanced stage disease without MYCN-amplification). However, based on mappings to the Unigene database, the number of UniGene clusters represented on each of the four microarray platforms used in the studies of Wei et al., Schramm et al., Ohira et al. and Oberthuer et al. only sums up to 362 UniGene clusters. In addition, the data of Wei et al. is not available in a public microarray data repository. Thus, we decided to use only data from the studies of Ohira et al. and Oberthuer et al.

In the study of Ohira et al. [7] a cDNA microarray platform with 5,430 probes representing 4,204 different GenBank entries was used. In the study of Oberthuer et al. [8] an oligonucleotide array with 10,163 probes representing 8,155 UniGene clusters was used. By means of sequence comparison, we found 1,271 genes that are represented on both platforms. Applying a minimum follow-up time of five years after initial diagnosis, gene expression profiles of MYCN non-amplified tumours with advanced INSS stage ( 3 or 4 ) from 66 patients were obtained in total from both studies (Table 1).

Although risk stratification and treatment strategies of neuroblastoma are different for a small fraction of patients [ref. [3] and Additional File 1] between Japan and Germany, the resulting 5-years overall survival is comparable among the German and Japanese neuroblastoma risk groups, suggesting that the selected patient cohorts from both studies $[7,8]$ can be compared in a combined analysis as performed here.

\section{Identification of significant genes}

To detect significant differential expression of a gene between the two outcome patient groups across studies,
Table I: Patient characteristics.

\begin{tabular}{ccc}
\hline Study & Oberthuer et al. & Ohira et al. \\
\hline $\begin{array}{c}\text { Outcome } \\
\text { F }\end{array}$ & 27 & 14 \\
UF & 16 & 9 \\
Age & & \\
$<$ Ia & 31 & 9 \\
$>$ Ia & 12 & 14 \\
Stage & & \\
3 & 13 & 13 \\
4 & 30 & 10 \\
Ip & & \\
Het & & \\
Im & 31 & n.d. \\
Del & 5 & n.d. \\
ND & 6 & n.d. \\
\hline
\end{tabular}

For the study of Ohira et al. no information about the Ip status was available(n.d.: not determined). F: favourable; UF: unfavourable; Het: heterozygous; Im: imbalance; Del: deletion; ND: not defined.

we applied a meta-analysis approach as described by Choi et al. [10]. Based on a $Q$ statistic it was decided to use a random effects model in the meta-analysis rather than a fixed effects model (Additional File 1). Using a random effects model and a threshold of 2.57 (corresponding to $\mathrm{p}$ $=0.01$ of a $\mathrm{N}(0,1)$ distribution) for the average effect size calculated on both data sets, we identified 72 genes which are significantly differentially expressed between the two outcome groups at a false discovery rate of $8.33 \%$. A number of 34 of these were found exclusively in the metaanalysis of both sets, and not in any single study databased analysis applying the same threshold (Table 2). This finding points to the fact that meta-analyses, due to an enhanced statistical power, may disclose differentially expressed genes that are missed by analyses of single study gene expression data sets.

To visualise the expression of the significant genes, colour maps were generated showing a hierarchical clustering of the gene expression values for each study separately (Fig. $1 \mathrm{~A}, \mathrm{~B})$. The samples of the study of Oberthuer et al. (Figure $1 \mathrm{~A}$ ) are grouped into threee main groups, as indicated by the sample dendrogram generated by hierarchical clustering. These three groups consist of the following samples (from left to right): NB412-NB331, NB279-NB025, NB572-NB250. The two leftmost groups mainly contain patients with unfavourable outcome, while only seven of 23 patients in these two groups show a favourable outcome. Of these seven patients, three already had a clinical event (see following paragraph about clinical courses of these patients). The third group (NB572-NB250) contains 20 patients, all having a favourable outcome. In the clustering results of Ohira et al., two samples can be identified that show an expression profile clearly distinct from the 
Table 2: List of differentially expressed genes.

\begin{tabular}{|c|c|c|c|}
\hline Symbol & Name & z score & only found in M.A. \\
\hline NDUFVI & NADH dehydrogenase (ubiquinone) flavoprotein I, $5 \mathrm{I} \mathrm{kDa}$ & -3.98 & \\
\hline TP53 & Tumor protein p53 (Li-Fraumeni syndrome) & -3.54 & \\
\hline $\mathrm{AHCY}$ & S-adenosylhomocysteine hydrolase & -3.44 & \\
\hline NPMI & Nucleophosmin (nucleolar phosphoprotein B23, numatrin) & -3.38 & $*$ \\
\hline CHDIL & Chromodomain helicase DNA binding protein I-like & -3.26 & \\
\hline CCNBI & Cyclin BI & -3.22 & \\
\hline HSPA5 & Heat shock 70 kDa protein 5 (glucose-regulated protein, $78 \mathrm{kDa}$ ) & -3.20 & \\
\hline SF4 & Splicing factor 4 & -3.19 & \\
\hline CCNA2 & Cyclin A2 & -3.15 & \\
\hline RPSI3 & Ribosomal protein SI3 & -3.14 & $*$ \\
\hline $\mathrm{E} 2 \mathrm{FI}$ & E2F transcription factor I & -3.12 & \\
\hline FLJII806 & Nuclear protein UKp68 & -3.12 & \\
\hline RUVBLI & RuvB-like I (E. coli) & -3.10 & \\
\hline ССТ5 & Chaperonin containing TCPI, subunit 5 (epsilon) & -3.05 & * \\
\hline ССТ3 & Chaperonin containing TCPI, subunit 3 (gamma) & -3.02 & \\
\hline DDX49 & DEAD (Asp-Glu-Ala-Asp) box polypeptide 49 & -2.89 & $*$ \\
\hline EIF2SI & Eukaryotic translation initiation factor 2 , subunit I alpha, $35 \mathrm{kDa}$ & -2.88 & \\
\hline KIFCI & Kinesin family member $\mathrm{Cl}$ & -2.88 & $*$ \\
\hline FLJ 13910 & Hypothetical protein FLJI3910 & -2.85 & \\
\hline APEXI & APEX nuclease (multifunctional DNA repair enzyme) I & -2.83 & \\
\hline COPB & Coatomer protein complex, subunit beta & -2.83 & \\
\hline CDC25B & Cell division cycle $25 \mathrm{~B}$ & -2.81 & $*$ \\
\hline TMED2 & transmembrane emp24 domain trafficking protein 2 & -2.73 & \\
\hline AHCYLI & S-adenosylhomocysteine hydrolase-like I & $-2.7 \mid$ & $*$ \\
\hline RAD23A & RAD23 homolog A (S. cerevisiae) & -2.70 & $*$ \\
\hline HMGB2 & High-mobility group box 2 & -2.67 & \\
\hline TRIM28 & Tripartite motif-containing 28 & -2.64 & $*$ \\
\hline ENOI & Enolase I, (alpha) & -2.62 & $*$ \\
\hline MRPL3 & Mitochondrial ribosomal protein $\mathrm{L} 3$ & -2.61 & $*$ \\
\hline MARK2 & MAP/microtubule affinity-regulating kinase 2 & -2.61 & $*$ \\
\hline DDXI & DEAD (Asp-Glu-Ala-Asp) box polypeptide I & -2.59 & $*$ \\
\hline KIF22 & Kinesin family member 22 & -2.57 & \\
\hline MLL & myeloid/lymphoid or mixed-lineage leukemia (trithorax homolog, Drosophila) & 2.57 & $*$ \\
\hline YWHAE & Tyrosine 3-monooxygenase/tryptophan 5-monooxygenase activation protein & 2.58 & \\
\hline DUSPI6 & Dual specificity phosphatase 16 & 2.62 & $*$ \\
\hline $\mathrm{EZHI}$ & Enhancer of zeste homolog I (Drosophila) & 2.63 & $*$ \\
\hline NCAMI & Neural cell adhesion molecule I & 2.63 & $*$ \\
\hline MAP2K4 & Mitogen-activated protein kinase kinase 4 & 2.64 & \\
\hline ELAVL4 & ELAV (embryonic lethal, abnormal vision, Drosophila)-like 4 (Hu antigen D) & 2.65 & $*$ \\
\hline RBMS3 & RNA binding motif, single stranded interacting protein & 2.65 & $*$ \\
\hline PXK & PX domain containing serine/threonine kinase & 2.66 & $*$ \\
\hline PMSCL2 & Exosome component 10 & 2.67 & $*$ \\
\hline NTRKI & Neurotrophic tyrosine kinase, receptor, type I & 2.67 & $*$ \\
\hline DOCK 4 & Dedicator of cytokinesis 4 & 2.70 & $*$ \\
\hline AB05I522 & DIX domain containing I & 2.70 & $*$ \\
\hline RAB2 & RAB2, member RAS oncogene family & 2.70 & $*$ \\
\hline FOXPI & Forkhead box PI & 2.74 & $*$ \\
\hline GPS2 & G protein pathway suppressor 2 & 2.79 & $*$ \\
\hline MLL5 & Myeloid/lymphoid or mixed-lineage leukemia 5 (trithorax homolog, Drosophila) & 2.82 & \\
\hline DLG4 & Discs, large homolog 4 (Drosophila) & 2.84 & $*$ \\
\hline VAMP2 & Vesicle-associated membrane protein 2 (synaptobrevin 2) & 2.85 & \\
\hline $\mathrm{NXPHI}$ & Neurexophilin I & 2.89 & $*$ \\
\hline ATP6VIA & ATPase, $\mathrm{H}+$ transporting, lysosomal $70 \mathrm{kDa}, \mathrm{VI}$ subunit $\mathrm{A}$ & 2.91 & \\
\hline ZNF2I8 & zinc finger protein 218 & 2.93 & \\
\hline FLJ 131110 & Receptor accessory protein I & 2.94 & \\
\hline CDK5RI & Cyclin-dependent kinase 5, regulatory subunit I (p35) & 2.96 & $*$ \\
\hline NCOA7 & nuclear receptor coactivator 7 & 2.96 & $*$ \\
\hline FLJII730 & Chromosome I open reading frame 149 & 3.04 & * \\
\hline MARCKS & Myristoylated alanine-rich protein kinase $C$ substrate & 3.06 & $*$ \\
\hline CLSTN3 & Calsyntenin 3 & 3.08 & \\
\hline
\end{tabular}


Table 2: List of differentially expressed genes. (Continued)

\begin{tabular}{llll}
\hline CAMTAI & Calmodulin binding transcription activator I & 3.10 & $*$ \\
SCN3B & sodium channel, voltage-gated, type III, beta & 3.1 I & 3.14 \\
PTN & Pleiotrophin (heparin binding growth factor 8, neurite growth-promoting factor I) & 3.26 & 3.26 \\
EPSI5 & Epidermal growth factor receptor pathway substrate I5 & 3.30 \\
BRUNOL4 & Bruno-like 4, RNA binding protein (Drosophila) & 3.38 \\
DCAMKLI & Doublecortin and CaM kinase-like I & 3.41 \\
ALS2CL & ALS2 C-terminal like & 3.42 \\
LOC284244 & Hypothetical protein LOC284244 & 3.50 \\
RAPGEF6 & Rap guanine nucleotide exchange factor (GEF) 6 & 3.89 \\
PKIB & Protein kinase (cAMP-dependent, catalytic) inhibitor beta & 3.96 \\
LOCII6236 & Hypothetical protein LOCII6236 &
\end{tabular}

Column 'only found in M.A.': Those genes are marked that were exclusively identified by meta-analysis. A negative z-score indicates down-regulation of a gene in tumors with a favourable prognosis relative to the expression in tumors with unfavourable prognosis.

other samples: S114 and S076. From the sample dendrogram and the heatmap, three groups can be derived for all remaining samples (from left to right): S091-S078, S108S080, S81-S90. The second group (S108-S080) mainly contains patients with unfavourable outcome (six out of eight), whereas the two other groups contain mainly patients with favourable outcome (11 of 13). Thus, most of the samples with similar clinical outcome are grouped together, with a better grouping resulting from the data of Oberthuer et al. Furthermore, the set of differentially expressed genes can also be divided into two groups according to their expression, one group shows low expression in tumours with favourable outcome and high expression in patients with an unfavourable outcome while the other group shows inverted expression behaviour. The gene dendrograms of the heatmaps in figure $1 \mathrm{~A}$ and $1 \mathrm{~B}$ both show a top level split that yields two roughly equal-sized groups of 32 and 40 genes (Fig. 1A) or 30 and 42 genes (Fig. 1B), respectively. The set of genes in these two groups are very similar in the two heatmaps for the different studies, as only two genes (TMED2 and MARK2) are grouped differently in the respective other study. Thus, the set of 72 can be divided into two groups in each of which genes show a highly consistant gene expression behaviour.

By generating further colour maps for the expression of the selected genes in MYCN amplified stage 3 or 4 tumours and MYCN non amplified tumours with stages 1 , 2, 4s of both studies (Fig. 2A,B), it was observed that the expression pattern of the genes selected by meta-analysis of intermediate risk neuroblastoma explicitly differs in the most aggressive tumours compared to tumours with very good prognosis. The full set of tumours from both studies is shown in Additional Files 2 and 3. The tumours with clearly defined prognosis show similar gene expression patterns for the set of 72 differentially expressed genes as the investigated tumour subgroup. These results suggest that all MYCN non-amplified tumours with advanced stage ( 3 or 4 ) can be distinguished into just two biological subtypes with contrasting clinical outcome according to the expression profile of the 72 differentially expressed genes. Moreover, expression of these genes also seems to be a marker for tumour aggressiveness in other tumour subgroups.

\section{Clinical courses observed in non-amplified advanced stage patients with differing gene-expression patterns}

In the data set of Oberthuer et al., seven patients that were alive five years after diagnosis were clustered together with those patients that had succumbed to disease (Fig. 1A). Three of them had experienced an event, two of them even had been treated with intensive salvage therapy, but all are currently in complete or incomplete remission. Opposed to that, three patients were documented to have experienced an event within the subset of patients that were clustered together as being alive five years after diagnosis. In all these patients, local tumour progression was diagnosed but either surgical intervention revealed maturation of the tissue or medium-dose chemotherapy resulted in event-free survival since then. Thus, a different quality of events was observed in those patients whose geneexpression profile resembles that of patients who succumbed to disease, as opposed to those events that were observed in patients whose gene-expression pattern matched long-term event-free survivors. For the data set of Ohira et al. (Fig. 1B) no detailed information about clinical history of investigated patients was available.

\section{Biological roles of the differentially expressed genes}

Using the set of 72 significant genes for a hierarchical clustering of MYCN amplified stage 3 or 4 tumours and MYCN non-amplified tumours with stages 1, 2, 4s, both prognosis groups are clearly separated (Fig. 2). Therefore, the expression of these 72 genes seems to be of general importance for distinction of prognosis groups in neuroblastoma tumours.

In the group of genes that shows high expression in tumours with favourable outcome, genes related to cell 
A

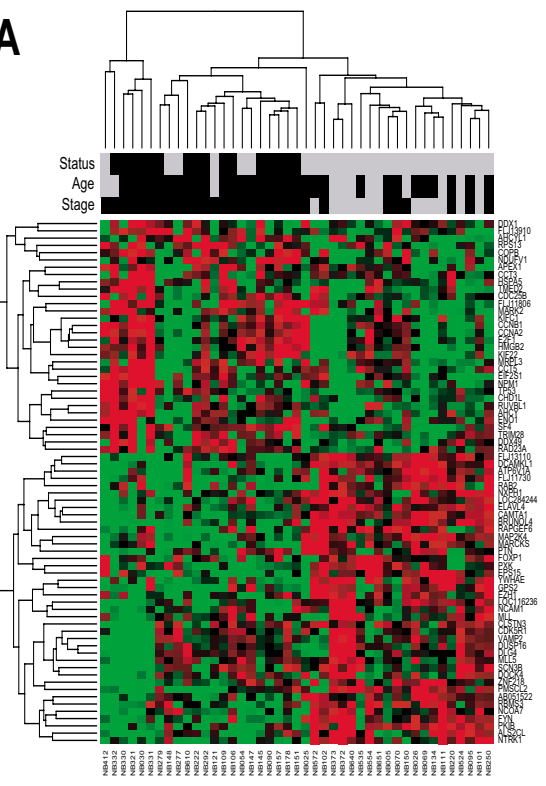

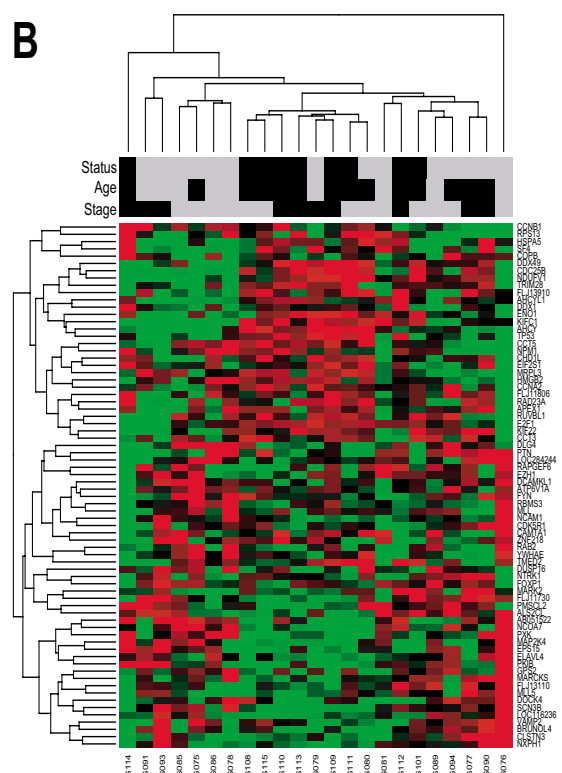

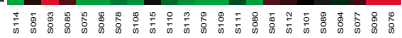

Figure I

Hierarchical clustering of the expression data for the significant genes in advanced stage MYCN non-amplified tumors. (A) Oligonucleotide data. (B) cDNA data. The colored bars at the top of the figure denote the values of the following clinical variables: Status: black - unfavourable outcome, grey - favourable outcome; Age (at diagnosis): black - older than one year, grey - younger than one year; Stage: black - INSS stage 4, grey - INSS stage 3.
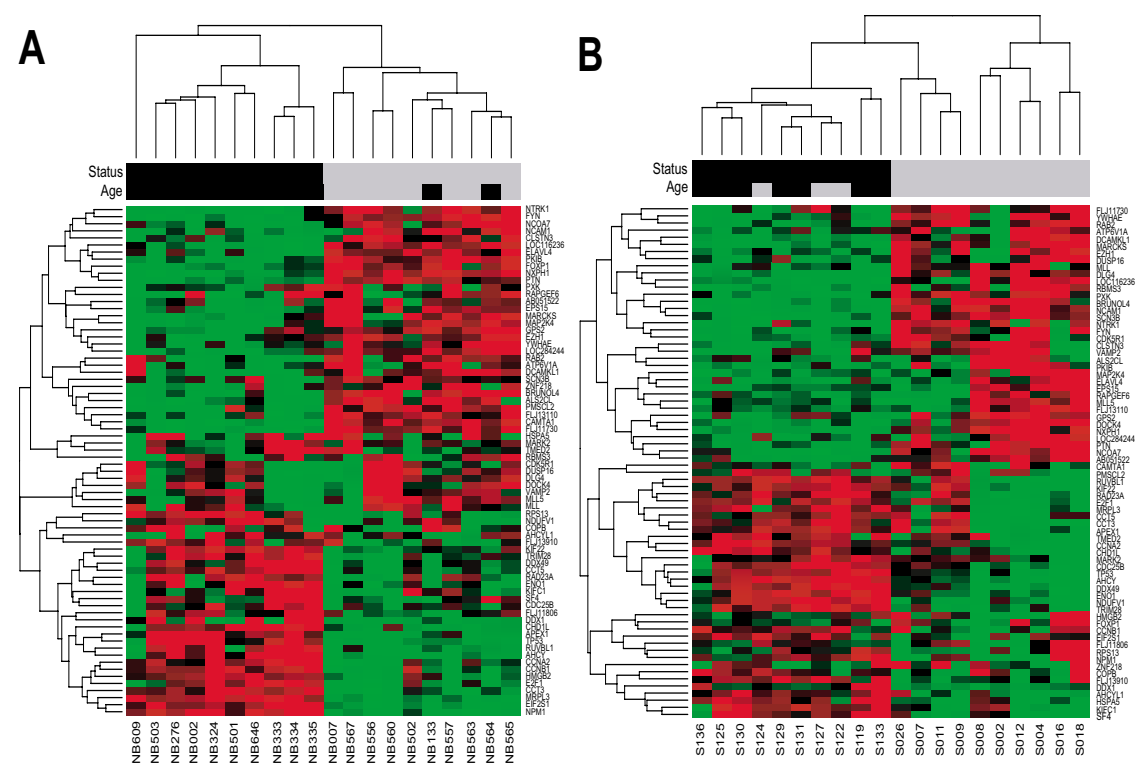

Figure 2

Hierarchical clustering of the expression data for the significant genes in advanced stage MYCN amplified tumors versus low stage non-amplified tumors. (A) Oligonucleotide data. (B) cDNA data. The colored bars at the top of the figure denote the values of the following clinical variables: Status: black - unfavourable outcome, grey - favourable outcome; Age (at diagnosis): black - older than one year, grey - younger than one year. 
Table 3: Enriched GO terms in the category "Biological Process" among the significantly differentially expressed genes.

\begin{tabular}{|c|c|c|c|c|c|}
\hline GO term & Expression & Count $\{55\}$ & Total $\{857\}$ & p-value & Corrected $p$ \\
\hline base-excision repair & - & 2 & 2 & 0.004 & 0.307 \\
\hline regulation of kinase activity & + & 5 & 19 & 0.005 & 0.307 \\
\hline regulation of protein kinase activity & + & 5 & 19 & 0.005 & 0.307 \\
\hline regulation of transferase activity & + & 5 & 20 & 0.007 & 0.307 \\
\hline negative regulation of transferase activity & + & 3 & 7 & 0.007 & 0.307 \\
\hline negative regulation of protein kinase activity & + & 3 & 7 & 0.007 & 0.307 \\
\hline negative regulation of enzyme activity & + & 3 & 8 & 0.011 & 0.361 \\
\hline regulation of enzyme activity & + & 5 & 25 & 0.018 & 0.361 \\
\hline response to DNA damage stimulus & - & 4 & 17 & 0.019 & 0.361 \\
\hline response to endogenous stimulus & - & 4 & 17 & 0.019 & 0.361 \\
\hline MAPKKK cascade & + & 3 & 10 & 0.022 & 0.361 \\
\hline one-carbon compound metabolism & - & 2 & 4 & 0.022 & 0.361 \\
\hline negative regulation of MAPK activity & + & 2 & 4 & 0.022 & 0.361 \\
\hline inactivation of MAPK activity & + & 2 & 4 & 0.022 & 0.361 \\
\hline M phase of mitotic cell cycle & - & 4 & 18 & 0.024 & 0.361 \\
\hline phosphorus metabolism & + & 9 & 68 & 0.033 & 0.361 \\
\hline phosphate metabolism & + & 9 & 68 & 0.033 & 0.361 \\
\hline$M$ phase & - & 4 & 20 & 0.034 & 0.361 \\
\hline regulation of cyclin dependent protein kinase activity & - & 2 & 5 & 0.036 & 0.361 \\
\hline glycolysis & - & 2 & 5 & 0.036 & 0.361 \\
\hline Wnt receptor signaling pathway & + & 2 & 5 & 0.036 & 0.361 \\
\hline oxidative phosphorylation & * & 2 & 5 & 0.036 & 0.361 \\
\hline
\end{tabular}

Expression: expression of associated genes in tumors with a favourable prognosis relative to the expression in tumors with unfavourable prognosis; +: up-regulation; -: down-regulation; *: genes show oppositional expression.

Count: number of significant genes associated with given GO term. Total: number of genes in analysis associated with given GO term. The numbers in curly brackets denote the number of genes that could be mapped to GO. Corrected p-values were calculated according to Benjamini and Hochberg as implemented in GOstat.

differentiation (FYN, NTRK1) and neuronal development (DCAMKL1) can be found. Among the significant genes that show a high expression in tumours with unfavourable outcome, cell cycle associated genes can be found (E2F1, CCNA2, CCNB1, KIFC1).

To further characterize which biological functions are defined by these genes, we explored the GO terms associated with the significant genes in order to identify the biological roles represented by these genes. We ranked the GO terms associated with the selected genes in our metaanalysis by using the p-values generated in a GO based gene set enrichment analysis as implemented in the GOstat tool. The most highly ranked annotation terms are listed in Table 3. Among these, GO terms can be found for which the associated genes all show higher expression in tumours with unfavourable outcome (e.g. cell cycle and DNA maintenance associated terms), as well as terms for which the associated genes show higher expression in tumours with favourable outcome (e.g. negative regulation of MAPK activity). The genes associated with the given GO terms are listed in Additional File 4.

\section{Classification analysis}

According to the German neuroblastoma risk stratification protocol, patients with non-MYCN amplified advanced stage 3 or 4 disease may be grouped into either the low-, the intermediate- or the high-risk group,

Table 4: Performance of different markers for outcome prediction on the independent test set.

\begin{tabular}{lccc}
\hline & Accuracy & Sensitivity & Specificity \\
\hline Microarray classifier & $0.77,[0.66 ; 0.85],(23 / 30)$ & $0.86,[0.62 ; 0.96],(6 / 7)$ & $0.74,[0.61 ; 0.84],(17 / 23)$ \\
Age & $0.53,[0.42 ; 0.65],(16 / 30)$ & $1.0,[0.81 ; 1.0],(7 / 7)$ & $0.39,[0.27 ; 0.52],(9 / 23)$ \\
Stage & $0.60,[0.48 ; 0.71],(18 / 30)$ & $1.0,[0.81 ; 1.0],(7 / 7)$ & $0.48,[0.35 ; 0.61],(11 / 23)$ \\
Age + Stage & $0.70,[0.58 ; 0.79],(21 / 30)$ & $1.0,[0.81 ; 1.0],(7 / 7)$ & $0.61,[0.48 ; 0.73],(14 / 23)$ \\
\hline
\end{tabular}

The figures in brackets denote $95 \%$ confidence intervals and absolute number of cases. For prediction of outcome by age at diagnosis, patients older than one year were predicted with unfavourable outcome. For prediction by stage, patients with stage 3 were predicted with favourable outcome and patients with stage 4 were predicted with unfavourable outcome. For the combination of the markers age and stage, only stage 4 patients older than one year at dignosis were predicted with unfavourable outcome. Sensitivity/Specificity is rate of correctly predicted patients with unfavourable/ favourable outcome. 
depending on their age at diagnosis, or an alteration of the short arm of chromosome 1 (del1p). Therefore, the investigated subgroup of neuroblastoma patients would greatly profit from an improved risk estimation. Independently to the analysis described above, we generated a predictive model for patient outcome using the method of nearest shrunken centroids classification [18] based on data of both studies. For this, 36 patients were selected from both studies as a training set, the remainig patients were used as a independent test set. The resulting predictive model, achieved a predictive accuracy of $77 \%$ for neuroblastoma in stage 3 or 4 without MYCN amplification in the independent test set (Table 4), using the expression values of 256 genes. Inferior predictive accuracies were achieved when the samples were classified by the clinical parameters age and stage, although no statistical significance of the difference in predictive accuracy between the best clinical parameter (a combination of age and stage) and the microarray based classification was observed, as the 95\% confidence intervals were overlapping. Application of the microarray based classifier also resulted in good performance in terms of both sensitivity and specificity, indicating the feasibility of generating and applying a predictive model of outcome based on data of different gene expression profiling studies. The overlap of the list of 256 genes used for classification with the list of 72 genes identified by meta analysis and the list of 144 genes used for classification in [8] is shown in Additional File 5.

\section{Discussion}

In this study, we combined gene expression data from two different studies to investigate the differences in gene expression for advanced stage MYCN non-amplified tumours with contrasting outcome at five years after initial diagnosis.

Our results suggest that this subgroup of tumours can be distinguished into two biological subtypes showing distinct gene expression profiles that are associated with contrasting clinical outcomes. The expression of the genes that are differentially expressed between these two subtypes may represent a general indicator of neuroblastoma aggressiveness, since corresponding expression behaviour can be observed in low stage MYCN non-amplified tumours as well as advanced stage MYCN amplified tumours.

Instead of simply comparing lists of differentially expressed genes obtained on single study data or combining p-values calculated for each single study [19], we applied a method of meta-analysis on the gene expression data that is based on a well established statistical framework and comprises modelling of study-to-study differences [10]. Unfortunately, the combination of gene expression data from different studies has the disadvan- tage that only genes common to all microarray platforms can be used. As the reliability of the probe mapping is crucial for a cross-platform analysis, we applied a stringent sequence based mapping of the probes of different microarray platforms in order to avoid inappropriate mapping of the probes. The combination of data from different studies for our analysis resulted in a large number of included expression profiles for the investigated subset of patients although a stringent selection criterion of a follow up of 5 years was applied. This clearly led to a higher statistical power of our analysis in comparison to single study based results, since of the 72 significantly differentially expressed genes, 34 genes were found exclusively in the meta-analysis of both sets.

Among the significantly differentially expressed genes are some that are known in the context of neuroblastoma research. Our results confirm observations made for these genes as described earlier in literature. High expression of NTRK1 is present in neuroblastomas with favourable biological features and highly correlated with patient survival [20]. High expression of FYN and high FYN kinase activity are restricted to low-stage tumours [21]. PTN is highly expressed in favourable neuroblastomas, whereas it is expressed at a significantly lower level in advanced tumours [22]. Low CAMTA1 expression is associated with poor outcome [23]. NCAM expression seems to enhance the malignancy of neuroblastoma cells and their tendency to metastasise [24]. High HuD (ELAVL4) mRNA levels may predict a clinically favourable outcome [25]. The fact that some of these genes were exclusively detected by the meta-analysis (Table 2) underlines the benefit of crossstudy analyses for investigation of tumour subgroups. Interestingly, the 72 genes found to be significant for the investigated subgroup of neuroblastoma tumours also show a distinct differential expression in other prognostic subgroups and may thus be used as a general prognostic marker for neuroblastoma patients. Moreover, this result suggests that, although for neuroblastoma tumours several different clinical stages and risk groups are defined, on the level of gene expression they seem to comprise only two distinct biological entities associated with adverse patient outcome.

The GO based gene set enrichment analysis of the GO terms associated with the selected genes in our meta-analysis did not show that any GO term is overrepresented with high statistical significance. However, the p-values calculated in the gene set enrichment analysis provide a useful ranking of the GO annotation terms that we used to select the genes shown in table 3 for characterization of the biological functions represented by the selected genes. Among the GO annotation terms associated with the genes selected in our meta-analysis, the GO-based gene set enrichment analysis highly ranked the cell cycle associ- 
ated GO terms. The up-regulation of the expression of cell cycle genes in aggressive neuroblastoma tumours was already observed by Krasnoselsky et al. [26] in a comparison of tumours of different stages and MYCN amplification status, where patient outcome was not regarded. In addition to the cell cycle genes, gene set enrichment analysis of the GO terms associated with the significant genes resulted in high rankings of three other GO terms known to be affected in tumorigenesis: DNA damage response, negative regulation of MAPK activity [27] and Wnt receptor signalling pathway [28]. For DNA-damage response genes, higher expression can be observed in tumours with a unfavourable outcome than in tumours with favourable outcome. This effect can also be seen in other tumour entities like prostate cancer. For the gene APEX1, gene expression data is available in the gene expression data repository Oncomine [29] that shows increasing expression according to tumour malignancy (Additional File 1). This effect might be caused by accumulated genetic abberations in tumours with unfavourable outcome which trigger the activity of DNA-damage response genes.

While interpreting the analysis of gene expression for non-amplified advanced stage neuroblastoma tumours with regard to patient outcome, the influence of the therapy that all these patients have received has to be taken into account, as differences of the gene expression profiles with regard to patient outcome may not only reflect tumour malignancy but also tumour responsiveness to the therapy.

Although only a small number of patients were available for generation and assessment of a predictive model, outcome prediction based on data of both studies (only genes common to both platforms) yielded good results. Both patients with favourable and unfavourable outcome were classified with good results as indicated by a balanced ratio of sensitivity and specificity. This shows the potential of the approach to use data of different gene expression studies to derive predictive models for patient subgroups where gene expression data is rare. However, for stable and compact (in terms of the number of used genes) predictive models, a larger total number of samples is needed [30] which could be realised by combination of future gene expression profiling studies with the approach used here.

\section{Conclusion}

In conclusion, cross-study analysis of gene expression data enables to detect consistent effects of gene expression based on completely independent data sets. The increase of statistical power through cross-study analysis is especially beneficial for the analysis of important patient subgroups for which gene expression data is rare. The presented results characterise a patient outcome specific set of differentially expressed genes in MYCN non-amplified advanced stage tumours. The resemblance of the expression of these genes in the investigated subgroup and in tumours with clearly defined (and diverging) outcome suggests that neuroblastomas comprise only two distinct biological subtypes associated with contrasting patient outcome.

Further investigations are needed to extend and validate these findings, a process in which the combination of future gene expression profiling studies which are larger both with respect to the investigated patients and probes on the utilised microarray platforms (thus yielding a large intersection of common probes), could play an important role.

\section{Competing interests}

The author(s) declare that they have no competing interests.

\section{Authors' contributions}

PW conceived of the study, carried out the analyses and drafted the manuscript. BB coordinated the study, participated in its design and helped to draft the manuscript. AO investigated the clinical records of the considered patients, contributed to the interpretation of the results and helped to draft the manuscript. MF and FW contributed to the interpretation of the results and helped to draft the manuscript. RE participated in the design of the study and helped to draft the manuscript. All authors read and approved the final manuscript.

\section{Additional material}

Additional File 1
Supplementary Information. Document giving further supplementary
information.
Click here for file
[http://www.biomedcentral.com/content/supplementary/1471-
$2407-7-89-S 1 . p d f]$
Additional File 2
Supplementary Figure 1 . Hierarchical clustering of the expression data for
the significant genes in advanced stage MYCN amplified tumors versus
low stage non-amplified tumors from the study of Oberthuer et al. [8]. All
samples with outcome information at 5 years after initial diagnosis have
been used. The colored bar at the top of the figure denotes the status of
outcome: black, fatal outcome; grey, favourable outcome. The colors refer
to high (red) or low (green) expression relative to gene-wise means. Genes
have been ordered by hierarchical clustering (correlation distance, com-
plete linkage algorithm; dendrogram not shown).
Click here for file
[http://www.biomedcentral.com/content/supplementary/1471-
$2407-7-89-S 2 . p d f]$

\section{Additional File 1}

lementary

[http://www.biomedcentral.com/content/supplementary/1471-

\section{Additional File 2} samples with outcome information at 5 years after initial diagnosis have been used. The colored bar at the top of the figure denotes the status of plete linkage algorithm; dendrogram not shown).

[http://www biomedcentral com/content/supplementary/1471 2407-7-89-S2.pdf] 


\section{Additional File 3 \\ Supplementary Figure 2. Hierarchical clustering of the expression data for the significant genes in advanced stage MYCN amplified tumors versus low stage non-amplified tumors from the study of Ohira et al. [7]. All samples with outcome information at 5 years after initial diagnosis have been used. The colored bar at the top of the figure denotes the status of outcome: black, fatal outcome; grey, favourable outcome. The colors refer to high (red) or low (green) expression relative to gene-wise means. Genes are shown in the same order as in Suppl. Fig. 1. \\ Click here for file \\ [http://www.biomedcentral.com/content/supplementary/1471- 2407-7-89-S3.pdf] \\ Additional File 4 \\ Supplementary Table 1. Genes associated with the GO categories men- tioned in Tab. 3 (main manuscript). \\ Click here for file \\ [http://www.biomedcentral.com/content/supplementary/1471- 2407-7-89-S4.xls] \\ Additional File 5 \\ Supplementary Figure 3. Venn diagram for comparison of the gene lists obtained by meta analysis ("Meta analysis"), used for cross-platform clas- sification ("CPC") or used for classification by Oberthuer et al. [8] ("Oberthür"). \\ Click here for file \\ [http://www.biomedcentral.com/content/supplementary/1471- 2407-7-89-S5.pdf]}

\section{Acknowledgements}

We thank Miki Ohira, Ph.D. and Akira Nakagawara, M.D., Ph.D. for provision of additional clinical data. PW receives a grant from the DFG Graduiertenkolleg 886 . The authors acknowledge financial support by the National Genome Research Network (0I GR 0450, 0 I GS 0457), by the Deutsche Krebshilfe (FKZ 50-27I9-Fi-I), and by the European Union's $6^{\text {th }}$ Framework Programme (LSHC-CT-2005-0189II).

\section{References}

I. Schwab M, Westermann F, Hero B, Berthold F: Neuroblastoma: biology and molecular and chromosomal pathology. Lancet Oncol 2003, 4:472-480.

2. Brodeur GM: Neuroblastoma: biological insights into a clinical enigma. Nat Rev Cancer 2003, 3:203-216.

3. Cheung NK, Cohn S: Neuroblastoma Berlin: Springer; 2005.

4. Maris JM: The biologic basis for neuroblastoma heterogeneity and risk stratification. Curr Opin Pediatr 2005, I7:7-I3.

5. Wei JS, Greer BT, Westermann F, Steinberg SM, Son C, Chen Q, Whiteford CC, Bilke S, Krasnoselsky AL, Cenacchi N, Catchpoole D, Berthold F, Schwab M, Khan J: Prediction of clinical outcome using gene expression profiling and artificial neural networks for patients with neuroblastoma. Cancer Res 2004, 64:6883-6891.

6. Schramm A, Schulte JH, Klein-Hitpass L, Havers W, Sieverts $H$, Berwanger B, Christiansen H, Warnat P, Brors B, Eils J, Eils R, Eggert A: Prediction of clinical outcome and biological characterization of neuroblastoma by expression profiling. Oncogene 2005, 24:7902-79|2

7. Ohira M, Oba S, Nakamura $Y$, Isogai E, Kaneko S, Nakagawa A, Hirata T, Kubo H, Goto T, Yamada S, Yoshida Y, Fuchioka M, Ishii S, Nakagawara $A$ : Expression profiling using a tumor-specific cDNA microarray predicts the prognosis of intermediate risk neuroblastomas. Cancer Cell 2005, 7:337-50.

8. Oberthuer A, Berthold F, Warnat P, Hero B, Kahlert $Y$, Spitz R, Ernestus K, König R, Haas S, Eils R, Schwab M, Brors B, Westermann
F, Fischer M: Customized oligonucleotide microarray gene expression-based classification of neuroblastoma patients outperforms current clinical risk stratification. Journal of Clinical Oncology 2006, 24:5070-8.

9. Ransohoff DF: Rules of evidence for cancer molecular-marker discovery and validation. Nat Rev Cancer 2004, 4:309-3I4.

10. Choi JK, Yu U, Kim S, Yoo OJ: Combining multiple microarray studies and modeling interstudy variation. Bioinformatics 2003 , I 9(SuppI I):i84-i90.

II. Warnat P, Eils R, Brors B: Cross-platform analysis of cancer microarray data improves gene expression based classification of phenotypes. BMC Bioinformatics 2005, 6:265.

12. Larkin JE, Frank BC, Gavras H, Sultana R, Quackenbush J: Independence and reproducibility across microarray platforms. Nat Methods 2005, 2:337-344.

13. Barrett T, Suzek TO, Troup DB, Wilhite SE, Ngau W, Ledoux P, Rudnev D, Lash AE, Fujibuchi W, Edgar R: NCBI GEO: mining millions of expression profiles-database and tools. Nucleic Acids Res 2005:D562-6.

14. Parkinson H, Sarkans U, Shojatalab M, Abeygunawardena N, Contrino S, Coulson R, Farne A, Lara GG, Holloway E, Kapushesky M, Lilja P, Mukherjee G, Oezcimen A, Rayner T, Rocca-Serra P, Sharma A, Sansone $S$, Brazma A: ArrayExpress-a public repository for microarray gene expression data at the EBI. Nucleic Acids Res 2005:D553-D555.

15. Gentleman RC, Carey V], Bates DM, Bolstad B, Dettling M, Dudoit S, Ellis B, Gautier L, Ge Y, Gentry J, Hornik K, Hothorn T, Huber W, lacus S, Irizarry R, Leisch F, Li C, Maechler M, Rossini AJ, Sawitzki G, Smith C, Smyth G, Tierney L, Yang JYH, Zhang J: Bioconductor: open software development for computational biology and bioinformatics. Genome Biol 2004, 5:R80.

16. Ashburner M, Ball CA, Blake JA, Botstein D, Butler H, Cherry JM, Davis AP, Dolinski K, Dwight SS, Eppig JT, Harris MA, Hill DP, IsselTarver L, Kasarskis A, Lewis S, Matese JC, Richardson JE, Ringwald M, Rubin GM, Sherlock G: Gene ontology: tool for the unification of biology. The Gene Ontology Consortium. Nat Genet 2000, 25:25-29.

17. Beissbarth T, Speed TP: GOstat: find statistically overrepresented Gene Ontologies within a group of genes. Bioinformatics 2004, 20: |464-|465.

18. Tibshirani R, Hastie T, Narasimhan B, Chu G: Diagnosis of multiple cancer types by shrunken centroids of gene expression. Proc Natl Acad Sci USA 2002, 99:6567-6572.

19. Rhodes DR, Barrette TR, Rubin MA, Ghosh D, Chinnaiyan AM: Meta-analysis of microarrays: interstudy validation of gene expression profiles reveals pathway dysregulation in prostate cancer. Cancer Res 2002, 62:4427-4433.

20. Nakagawara A, Arima-Nakagawara M, Scavarda NJ, Azar CG, Cantor $A B$, Brodeur GM: Association between high levels of expression of the TRK gene and favorable outcome in human neuroblastoma. N Engl J Med 1993, 328:847-854.

21. Berwanger B, Hartmann O, Bergmann E, Bernard S, Nielsen D, Krause M, Kartal A, Flynn D, Wiedemeyer R, Schwab M, Schäfer H, Christiansen $\mathrm{H}$, Eilers M: Loss of a FYN-regulated differentiation and growth arrest pathway in advanced stage neuroblastoma. Cancer Cell 2002, 2:377-386.

22. Nakagawara A, Milbrandt J, Muramatsu T, Deuel TF, Zhao H, Cnaan A, Brodeur GM: Differential expression of pleiotrophin and midkine in advanced neuroblastomas. Cancer Res 1995, 55: $1792-1797$.

23. Henrich K, Fischer M, Mertens D, Benner A, Wiedemeyer R, Brors B, Oberthuer A, Berthold F, Wei JS, Khan J, Schwab M, Westermann F: Reduced expression of CAMTAI correlates with adverse outcome in neuroblastoma patients. Clin Cancer Res 2006, 12:131-138.

24. Gluer S, Zense M, Radtke E, von Schweinitz D: Expression of polysialylated NCAM on neuroblastomas of various histology and clinical stages. Langenbecks Arch Chir Suppl Kongressbd 1998, I I 5(Suppl I):289-292.

25. Ball NS, King PH: Neuron-specific hel-NI and HuD as novel molecular markers of neuroblastoma: a correlation of HuD messenger RNA levels with favorable prognostic features. Clin Cancer Res 1997, 3:1859-1865.

26. Krasnoselsky AL, Whiteford CC, Wei JS, Bilke S, Westermann F, Chen Q, Khan J: Altered expression of cell cycle genes distin- 
guishes aggressive neuroblastoma. Oncogene 2005, 24:|533-I54I.

27. Freeman SM, Whartenby KA: The role of the mitogen-activated protein kinase cellular signaling pathway in tumor cell survival and apoptosis. Drug News Perspect 2004, 17:237-242.

28. Karim R, Tse G, Putti T, Scolyer R, Lee S: The significance of the Wnt pathway in the pathology of human cancers. Pathology 2004, 36:120-128.

29. Rhodes DR, Yu J, Shanker K, Deshpande N, Varambally R, Ghosh D, Barrette T, Pandey A, Chinnaiyan AM: ONCOMINE: a cancer microarray database and integrated data-mining platform. Neoplasia 2004, 6: I-6.

30. Ein-Dor L, Zuk O, Domany E: Thousands of samples are needed to generate a robust gene list for predicting outcome in cancer. Proc Natl Acad Sci USA 2006, 103:5923-8.

\section{Pre-publication history}

The pre-publication history for this paper can be accessed here:

http://www.biomedcentral.com/1471-2407/7/89/prepub

Publish with Biomed Central and every scientist can read your work free of charge

"BioMed Central will be the most significant development for disseminating the results of biomedical research in our lifetime. "

Sir Paul Nurse, Cancer Research UK

Your research papers will be:

- available free of charge to the entire biomedical community

- peer reviewed and published immediately upon acceptance

- cited in PubMed and archived on PubMed Central

- yours - you keep the copyright

Submit your manuscript here:

http://www.biomedcentral.com/info/publishing_adv.asp 